\title{
Fibras metálicas e sua influência no comportamento mecânico de concretos refratários durante a secagem
}

\section{(Influence of steel fiber on the mechanical behavior of refractory castables upon drying)}

\author{
C. M. Peret, V. C. Pandolfelli \\ Grupo de Engenharia de Microestrutura de Materiais - GEMM \\ Departamento de Engenharia de Materiais, Universidade Federal de S. Carlos \\ Rod. Washington Luiz, km 235, C.P. 676, S. Carlos, SP, 13565-905 \\ peret@iris.ufscar.br,vicpando@power.ufscar.br
}

\begin{abstract}
Resumo
A adição de fibras poliméricas pode auxiliar no aumento da resistência à explosão de concretos refratários por meio de dois mecanismos principais: aumento de permeabilidade ou reforço mecânico. Já as firas metálicas apresentam um potencial de reforço para a secagem, por manterem valores altos de resistência mecânica e de módulo de elasticidade na faixa de temperaturas crítica, entre 150 e $200{ }^{\circ} \mathrm{C}$. Neste trabalho, estudou-se o comportamento mecânico e de secagem de concretos contendo fibras metálicas curtas e longas, comparando-os com fibras poliaramídicas (PAr). As fibras metálicas aumentaram consideravelmente a energia de fratura, porém foram pouco eficientes na contenção do dano de secagem. O volume unitário dessas fibras, três ordens de grandeza superior ao da PAr, resulta em uma menor quantidade de fibras por unidade de volume. Por isso, solicitações mecânicas na matriz são menos beneficiadas pelo reforço gerado por essas fibras. Os resultados mostram a importância de considerar a escala das solicitações para definir a geometria dos elementos de reforço, a fim de otimizar seu desempenho.
\end{abstract}

Palavras-chave: energia total de fratura, resistência à explosão, concreto refratário, fibras metálicas, secagem.

Abstract

The addition of fibers to refractory castables can improve their dry-out behavior by two main mechanisms: permeability increase or mechanical reinforcement. Steel fibers present a great potential as reinforcement, because their tensile strength and modulus of elasticity are maintained even at high temperatures. In this work, the mechanical behavior and the resistance to spalling on the drying of a refractory castable containing short and long steel fibers (from 2 to $25 \mathrm{~mm}$ long) were evaluated, and compared to the behavior of the same castable with polyaramid (PAr) fibers. The steel fibers improved the work-of-fracture but were not efficient in avoiding damage upon drying. Their volume, about one thousand times that of the polymeric fibers, results in a lower number of fibers per unit volume of the castable. Therefore, localized stress fields in the matrix cannot have full benefit from the reinforcement provided by these fibers. The results attested the need of taking into account the magnitude of the expected stress fields in order to define the size and shape of the reinforcement particles that will lead to an optimum performance.

Keywords: work-of-fracture, spalling resistance, refractory castable, steel fibers, drying.

\section{INTRODUÇÃO}

A secagem de concretos refratários vem sendo intensamente pesquisada, devido à importância atribuída a essa etapa do processamento pelos fabricantes e usuários deste material. $\mathrm{O}$ desenvolvimento de formulações otimizadas do ponto de vista de secagem permite minimizar $\mathrm{o}$ risco de dano desse material durante o processamento e reduzir o tempo de parada de equipamentos em manutenção ou reparo, reduzindo o custo e aumentando a produtividade.

A utilização de fibras poliméricas tem-se mostrado um meio eficaz de aumentar a resistência do concreto ao dano por secagem, permitindo impor condições mais severas de aquecimento, sob menor risco.

Dois mecanismos foram identificados como responsáveis pelos benefícios conferidos pelas fibras poliméricas: 1) o aumento de permeabilidade proporcionado pela fusão, degradação ou retração das fibras [1], como no caso do polipropileno, e 2) o reforço mecânico, sobretudo pelo aumento da energia dissipada durante a propagação de trincas [2], como aquele proporcionado pela adição de fibras de poliaramida (PAr). 
No caso de fibras adicionadas ao concreto com o objetivo de proporcionar reforço mecânico, como a PAr, deve-se garantir que a fibra mantenha boas propriedades elásticas na faixa de temperatura em que o risco de dano é elevado. Para os concretos refratários com baixo teor de cimento, trabalhos anteriores [3] mostraram que essa faixa está geralmente situada entre 150 e $180{ }^{\circ} \mathrm{C}$. Dessa forma, a poliaramida, fibra polimérica que apresenta degradação térmica somente em temperaturas superiores a $300{ }^{\circ} \mathrm{C}$, mostrou-se eficaz na prevenção de explosão de um concreto refratário durante a secagem [2].

Além de polímeros, vários outros materiais têm sido utilizados com sucesso como reforço mecânico, na obtenção de compósitos com alta resistência à propagação de trincas. No caso da construção civil e da indústria refratarista, fibras de aço-carbono e inoxidável são tradicionalmente utilizadas em diversos tamanhos e geometrias, para reforço à temperatura ambiente e em altas temperaturas [3].

Baseando-se no sucesso das fibras metálicas como reforço mecânico em diversas aplicações, e na identificação desse mecanismo como influente na resistência ao dano por secagem, buscou-se, neste trabalho, caracterizar a eficiência de fibras metálicas curtas (de comprimento médio igual a 2, 4 e $6 \mathrm{~mm}$ ) e longas ( $25 \mathrm{~mm}$ de comprimento) na determinação da resistência ao dano por secagem.

\section{METODOLOGIA}

Foi desenvolvido um concreto refratário de alta alumina e ultra-baixo teor de cimento, segundo uma distribuição granulométrica de Andreasen com q = 0,24. Esse coeficiente foi escolhido por facilitar a obtenção de um material com boas características reológicas para a moldagem por vibração, além de baixa tendência à dilatância, o que é especialmente vantajoso na incorporação de fibras ao concreto, já que a presença de fibras com alta razão de aspecto (L/d) dificulta o empacotamento das partículas, favorecendo a geração de defeitos [4].

A Tabela I indica as matérias-primas utilizadas, e a

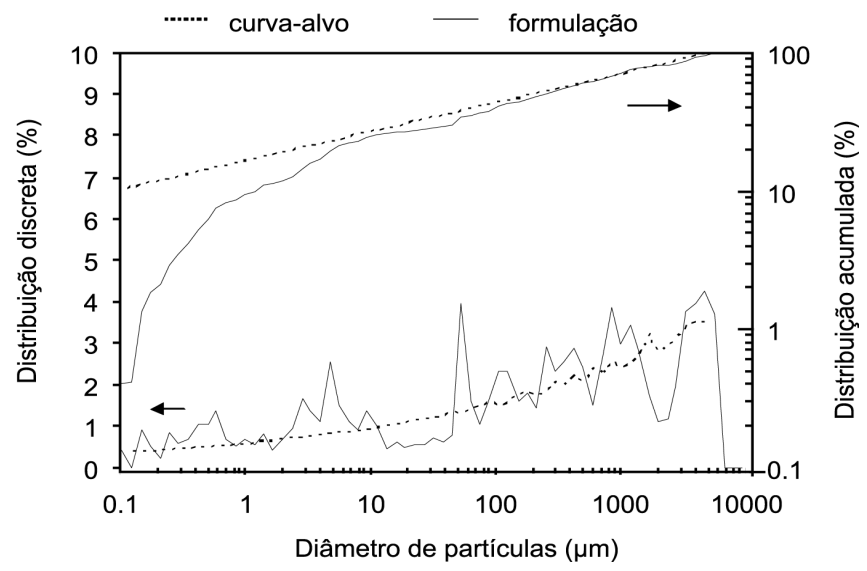

Figura 1: Curva-alvo (Andreasen) e a distribuição granulométrica resultante do concreto formulado.

[Figure 1: Target curve (Andreasen) and the actual particle size distribution of the castable.]

Fig. 1 apresenta a curva-alvo de Andreasen e exemplifica uma distribuição real resultante da combinação das matériasprimas disponíveis. Para a dispersão das partículas, utilizou-se ácido cítrico anidro, em um teor igual a $0,05 \%$ em peso.

Foram selecionadas quatro fibras metálicas com diferentes comprimentos: Gervois, Stax 130, Stax 225 e Daoli, fabricadas em aço inoxidável tipo AISI 410, com massa específica igual a $7,34 \mathrm{~g} / \mathrm{cm}^{3}$. As fibras Gervois e Stax foram fabricadas pela moagem de lã de aço e sua distribuição de comprimentos foi caracterizada por análise de imagem (Fig. 2). No caso da fibra Daoli, fabricada pelo processo Melt Extraction, o comprimento é de $25 \mathrm{~mm}$. Além das fibras metálicas, utilizouse para comparação as de poliaramida (PAr), uma vez que estas fibras também não se degradam na faixa de temperatura crítica para a secagem $\left(150-180^{\circ} \mathrm{C}\right)$.

O comprimento e diâmetro médios dessas fibras encontramse na Tabela II, juntamente com o número médio de fibras contidas em um centímetro cúbico do concreto. Este valor provém de um cálculo ideal, provavelmente superestimado devido à dispersão dos comprimentos reais das fibras. Em

Tabela I - Matérias-primas utilizadas no concreto refratário.

[Table I - Raw materials for the castable preparation.]

\begin{tabular}{lc}
\hline Matéria-prima & Teor em peso (\%) \\
\hline $\begin{array}{l}\text { Alumina eletrofundida branca } \\
\text { (frações 4/10, 8/20, 10/40, 20/40, 40/F, 60/F, 200/F) }\end{array}$ & 76 \\
\hline Alumina calcinada ${ }^{2}$ (A17-NE, A1000 SG) & 22 \\
\hline Cimento de Aluminato de Cálcio ${ }^{3}$ (CA-14 R) & 2 \\
\hline${ }^{1}$ Elfusa Eletrofusão S.A. ${ }^{2}$ Alcoa Brasil e Alemanha & ${ }^{3}$ Alcoa Alemanha
\end{tabular}




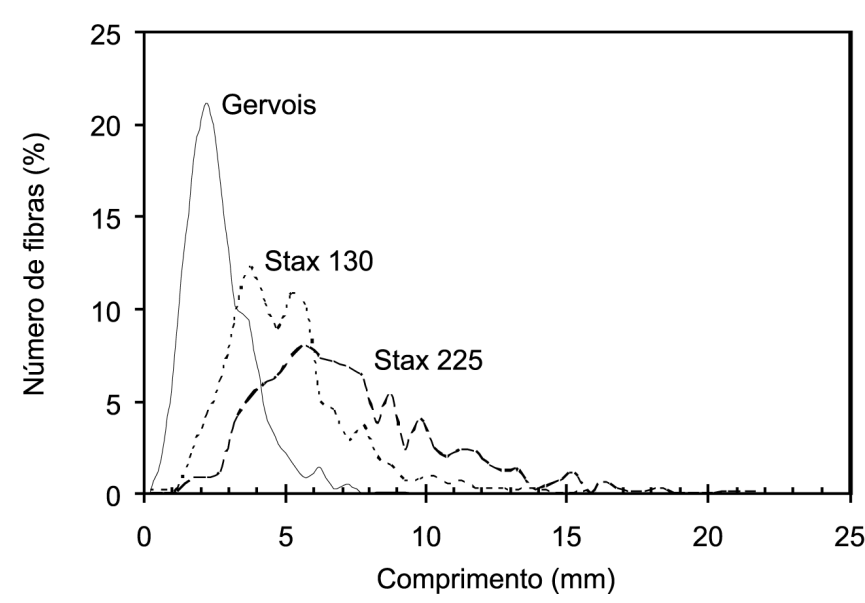

Figura 2: Distribuição de comprimentos das fibras metálicas curtas utilizadas.

[Figure 2: Length statistical distribution for the short steel fibers.]

todos os casos estudados, fixou-se o teor volumétrico das fibras adicionadas ao concreto em $0,36 \%$. A adição ocorreu sempre durante a mistura a seco, tomando-se cuidado para impedir a introdução de aglomerados de fibras.

A mistura a úmido foi realizada em um reômetro para concretos refratários, desenvolvido pelo Grupo de Engenharia de Microestrutura de Materiais - GEMM [5]. A quantidade de água utilizada foi mantida constante e igual a 4,5\% em peso do concreto ( $15 \%$ em volume). O método de adição da água influencia a eficiência do processo de mistura [6, 7], modificando as condições de cisalhamento entre as partículas. Neste estudo, a água foi introduzida seguindo-se o roteiro apresentado na Tabela III. Esse cuidado garantiu que as curvas de mistura de todas as composições fossem comparáveis entre si e que a dispersão das partículas fosse otimizada.

Foram moldados em mesa vibratória corpos cilíndricos de $40 \mathrm{~mm}$ de diâmetro por $40 \mathrm{~mm}$ de altura para compressão diametral e ensaios de secagem; corpos prismáticos de
25 x $25 \times 150 \mathrm{~mm}^{3}$ com entalhe pré-moldado de $1 \mathrm{~mm}$ de profundidade por $1 \mathrm{~mm}$ de espessura para ensaios de $\gamma_{\text {wof }}$; e corpos cilíndricos de $75 \mathrm{~mm}$ de diâmetro e $22 \mathrm{~mm}$ de altura para medida da permeabilidade a temperatura ambiente. Para garantir um bom preenchimento dos moldes, o concreto foi vibrado por cerca de 1 minuto durante a moldagem em mesa vibratória NTG.

Os corpos-de-prova foram curados por 7 dias, a $8{ }^{\circ} \mathrm{C}$, em ambiente saturado de água, com exceção das amostras para permeabilidade, que foram curadas por 7 dias, a $50{ }^{\circ} \mathrm{C}$, em ambiente saturado e em seguida secos por 3 dias a $50{ }^{\circ} \mathrm{C}$. A temperatura de $8{ }^{\circ} \mathrm{C}$ foi escolhida para a cura por levar à formação da fase hidratada $\mathrm{CAH}_{10}\left(\mathrm{CaO} \cdot \mathrm{Al}_{2} \mathrm{O}_{3} \cdot 10 \mathrm{H}_{2} \mathrm{O}\right)$, de alto volume molar. Com isso, a permeabilidade do material é reduzida, permitindo obter uma condição crítica para a secagem.

A caracterização mecânica dos compósitos foi realizada por meio de ensaios de compressão diametral (ASTM C496-96) e energia total de fratura, ambos à temperatura ambiente. Os ensaios foram realizados logo após a cura, sem uma etapa prévia de secagem, a fim de melhor reproduzir as propriedades do material quando ainda saturado de água.

A resistência do material à tração é obtida pela equação

$$
\sigma_{1}=\frac{2}{\pi} \cdot \frac{P}{d \cdot h}
$$

em que P é a carga de ruptura $(\mathrm{N})$, d é o diâmetro do corpode-prova (m) e h, sua altura (m).

A energia total de fratura, $\gamma_{\text {wof }}$, foi calculada pela integral da curva de força, P, em função do deslocamento do atuador, $d$, a partir de um ensaio de flexão a três pontos sob taxa de deslocamento constante $(20 \mu \mathrm{m} / \mathrm{min})$.

No caso da permeabilidade em temperatura ambiente (PBT), foram obtidos dados de vazão de ar através de uma amostra cilíndrica de $75 \mathrm{~mm}$ de diâmetro e $22 \mathrm{~mm}$ de

Tabela II - Comprimento, diâmetro e número médio de fibras por volume unitário de concreto. [Table II - Average length, diameter and number of fibers per unit volume of the castable.]

\begin{tabular}{|c|c|c|c|c|}
\hline & $\begin{array}{c}\% \text {-vol. } \\
\text { [\%-peso] }\end{array}$ & $\begin{array}{l}\text { Diâmetro } \\
(\mu \mathrm{m})\end{array}$ & Comprimento (mm) & $\begin{array}{l}\text { fibras } / \mathrm{cm}^{3} \\
(0,36 \%-\mathrm{vol})\end{array}$ \\
\hline Poliaramida $^{1}$ & $0,36[0,12]$ & 20 & 6 & 1900 \\
\hline Gervois $^{2}$ & $0,36[0,66]$ & 70 & 2 & 470 \\
\hline Stax $130^{3}$ & $0,36[0,66]$ & 130 & 4 & 70 \\
\hline Stax $225^{3}$ & $0,36[0,66]$ & 180 & 6 & 25 \\
\hline Daoli $^{4}$ & $0,36[0,66]$ & 450 & 25 & 0,9 \\
\hline
\end{tabular}


Tabela III - Roteiro de mistura para preparação do concreto. [Table III - Mix procedure for the castable preparation.]

\begin{tabular}{cc}
\hline Tempo de mistura & Evento \\
\hline $0 \mathrm{~s}$ & Início da mistura a seco \\
\hline $60 \mathrm{~s}$ & $\begin{array}{c}\text { Adição de água para virada } \\
(3,6 \% \text { - } \text {; vazão const.) }\end{array}$ \\
\hline $\begin{array}{c}\text { Momento da virada } \\
\text { (entre 150 e 170 s) }\end{array}$ & $\begin{array}{c}\text { Adição da água restante } \\
\text { (total de 4,5\%-p) }\end{array}$ \\
\hline 60 s após a virada & $\begin{array}{c}\text { Fim da mistura } \\
\text { (total = 210 a 230 s) }\end{array}$ \\
\hline
\end{tabular}

altura, em função da pressão de entrada de ar. Esses dados permitiram a obtenção das constantes $\mathrm{k}_{1} \mathrm{e} \mathrm{k}_{2}$, de acordo com a Equação de Forchheimer:

$$
\frac{\mathrm{P}_{\mathrm{i}}^{2}-\mathrm{P}_{0}^{2}}{2 \mathrm{P}_{\mathrm{O}} \mathrm{L}}=\frac{\mu}{\mathrm{k}_{1}} \mathrm{~V}_{\mathrm{S}}+\frac{\rho}{\mathrm{k}_{2}} \mathrm{~V}_{\mathrm{S}}^{2}
$$

onde $\mathrm{P}_{\mathrm{i}}$ e $\mathrm{P}_{0}$ são, respectivamente, as pressões de entrada e saída de ar no material, L é a altura do corpo-de-prova, $\mu$ e $\rho$ são a viscosidade e a densidade do ar, e $\mathrm{V}_{\mathrm{S}}$ é a velocidade de escoamento do ar no interior do material, calculada a partir da vazão medida e da área de seção transversal da amostra [8].

As medidas de PBT foram efetuadas em corpos-de-prova secos, ainda a verde, e em seguida nas mesmas amostras, após queima a $900{ }^{\circ} \mathrm{C}$, por $6 \mathrm{~h}$.

Para a verificação do comportamento de secagem, utilizouse um equipamento termogravimétrico construído no grupo de pesquisa dos autores [9]. Trata-se de um forno elétrico com capacidade para a implementação de taxas de aquecimento de até $20{ }^{\circ} \mathrm{C} / \mathrm{min}$, dentro do qual é alojado um porta-amostra conectado a uma balança. Para a proteção das resistências do forno, o porta-amostra é envolvido por uma gaiola metálica, evitando o lançamento de estilhaços oriundos da explosão do concreto, quando esta ocorrer. O corpo-de-prova é aquecido a $20^{\circ} \mathrm{C} / \mathrm{min}$, da temperatura ambiente até $600^{\circ} \mathrm{C}$. O ensaio pode ser acompanhado pelo parâmetro $\mathrm{W}(\mathrm{t})$, que representa uma medida da porcentagem cumulativa de água que já tenha saído do corpo no instante $t$, em função do total de água inicialmente presente:

$$
\mathrm{W}(\mathrm{t})=100 \mathrm{x}\left[\frac{\mathrm{m}_{0}-\mathrm{m}(\mathrm{t})}{\mathrm{m}_{0}-\mathrm{m}_{\mathrm{f}}}\right]
$$

sendo $m(t), m_{0}$ e $m_{f}$, respectivamente, a massa do corpo no instante t, sua massa inicial e a massa do corpo seco. Para a análise dos dados, calculou-se a taxa instantânea de secagem derivando-se $\mathrm{W}(\mathrm{t})$ em função do tempo, ponto a ponto.

\section{RESULTADOS E DISCUSSÃ̃}

As curvas de torque em função do tempo, referentes à mistura dos concretos desenvolvidos para este estudo são apresentadas na Fig. 3. A figura também mostra as diferenças observadas entre o concreto contendo fibras metálicas, aquele com fibras de poliaramida e o concreto sem fibras ("Ref."). Praticamente não houve alteração do formato das curvas pela adição de fibras metálicas, exceto uma antecipação de aproximadamente $13 \%$ no tempo para a virada nos concretos com fibras Stax 130 e 225, e um aumento próximo a $13 \%$ no torque final para todos os concretos contendo fibras metálicas. Estas alterações observadas são modestas, frente àquelas obtidas pela adição de fibras de poliaramida [10]. Neste caso, o torque atingiu valores cerca de $30 \%$ superiores ao obtido com o material de referência.

O esforço médio de mistura pode ser calculado pela integral das curvas de mistura mostradas na Fig. 3, e é um indicativo do trabalho realizado pelo equipamento durante todo o processo (Fig. 4). Nota-se um esforço superior para a mistura do concreto com poliaramida. Essas fibras poliméricas possuíam cerca de $6 \mathrm{~mm}$ de comprimento, portanto próximo a algumas das fibras metálicas estudadas, porém um diâmetro da ordem de $20 \mu \mathrm{m}$ (ver Tabela II).

De acordo com suas características geométricas, estimase que o número médio de fibras por centímetro cúbico de material, para o teor constante de $0,36 \%$-vol., seja de 470,70 , 25 e 0,9 fibras $/ \mathrm{cm}^{3}$ para as fibras Gervois, Stax 130, Stax 225 e Daoli, respectivamente. No entanto, para a poliaramida, esse valor sobe para cerca de 1900 fibras $/ \mathrm{cm}^{3}$ (Tabela II). Esses resultados indicam que o número de fibras presentes em um volume unitário de material é um fator importante no comportamento reológico do concreto durante a mistura e, provavelmente, durante o bombeamento.

A Fig. 5 apresenta a constante de permeabilidade $\mathrm{k}_{2}$, obtida pela Equação de Forchheimer [8] para os concretos

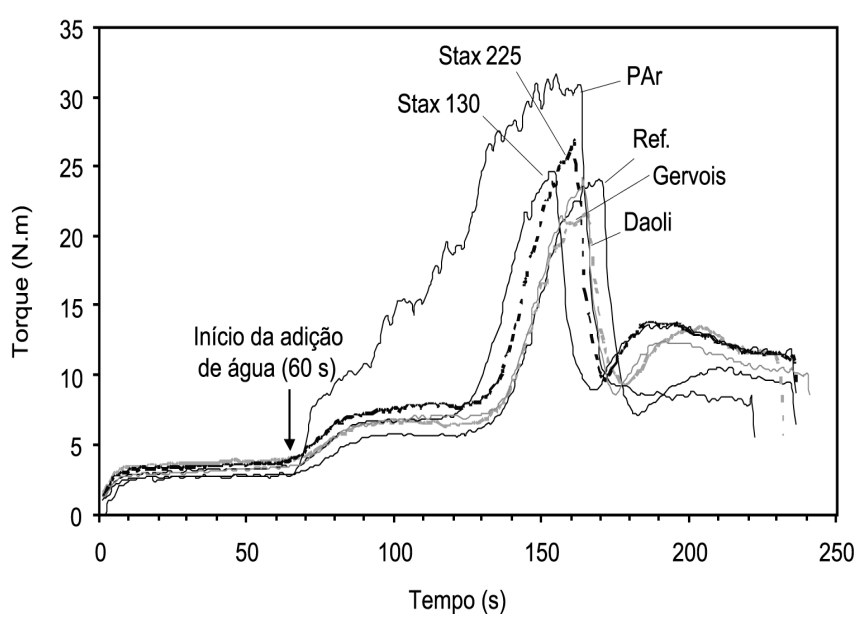

Figura 3: Curvas de mistura dos concretos refratários contendo fibras $(0,36 \%$-vol).

[Figure 3: Torque versus mixing time for the fiber-containing refractory castables.] 


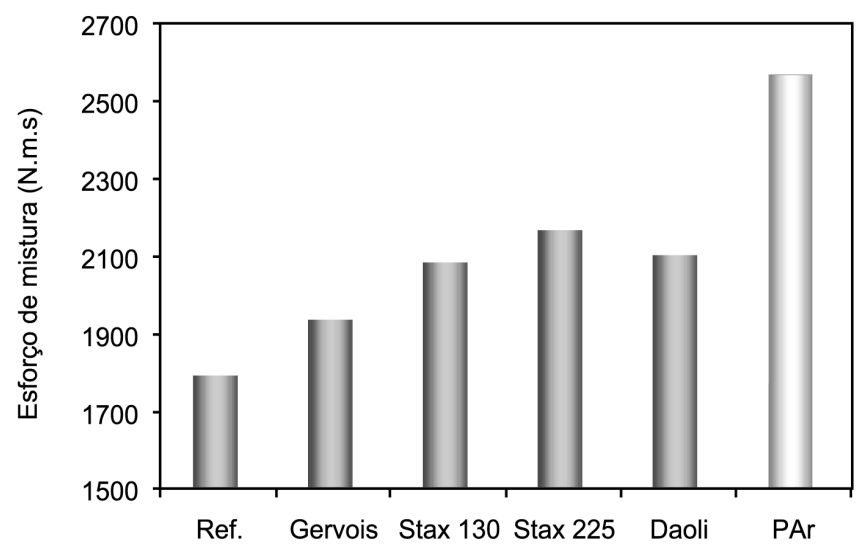

Figura 4: Esforço médio de mistura para os concretos contendo fibras metálicas.

[Figure 4: Average mixing effort for the fiber-containing castables.]

estudados. Não foi verificada diferença apreciável de permeabilidade entre os materiais a verde. Nos concretos queimados houve um aumento na permeabilidade em função do comprimento das fibras metálicas adicionadas (Gervois < Stax $130<$ Stax 225 < Daoli), possivelmente decorrente dos defeitos na interface fibra-matriz. A permeabilidade dos materiais queimados aumentou em todos os casos, e o aumento foi tanto maior quanto maiores as fibras metálicas adicionadas, devido às diferenças de coeficiente de dilatação térmica e o módulo de elasticidade entre o metal e a cerâmica. No caso da fibra de poliaramida, o aumento de permeabilidade foi uma ordem de grandeza superior ao ocorrido com as fibras metálicas, devido à degradação térmica e à geração de canais permeáveis finamente divididos na microestrutura do concreto [1].

A resistência dos concretos à compressão diametral variou entre 0,8 e 1,4 MPa, como mostra a Fig. 6. A fibra Daoli, mais longa entre as metálicas, proporcionou o maior aumento na resistência mecânica. A adição de poliaramida pouco alterou essa propriedade. Esse resultado concorda com a literatura, que mostra que os teores passíveis de serem adicionados ao

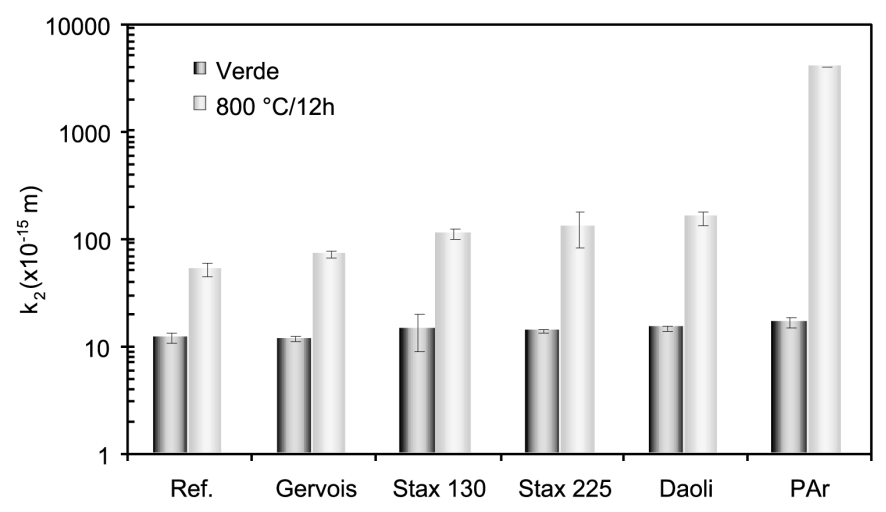

Figura 5: Constante de permeabilidade para o concreto refratário com as diferentes fibras estudadas.

[Figure 5: Permeability of the green and fired fiber-containing refractory castables.]

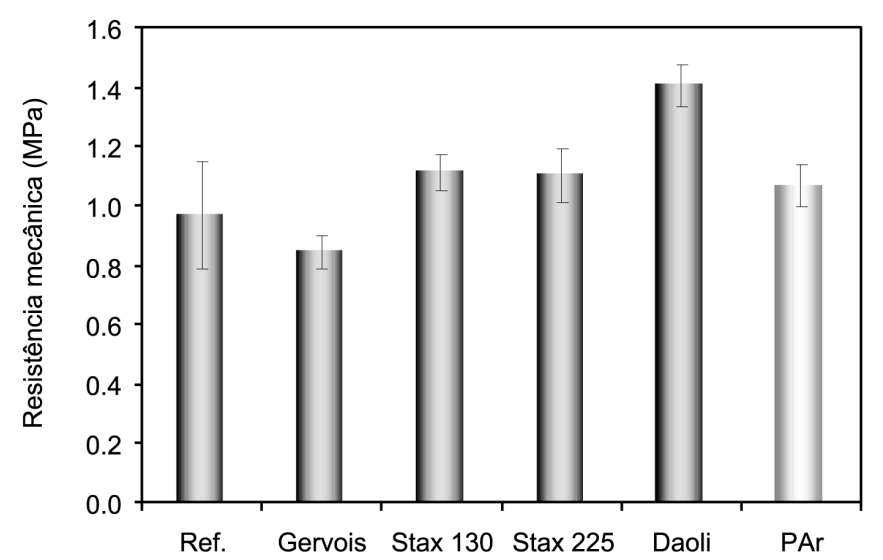

Figura 6: Resistência mecânica dos concretos por compressão diametral.

[Figure 6: Splitting tensile strength for the refractory castables.]

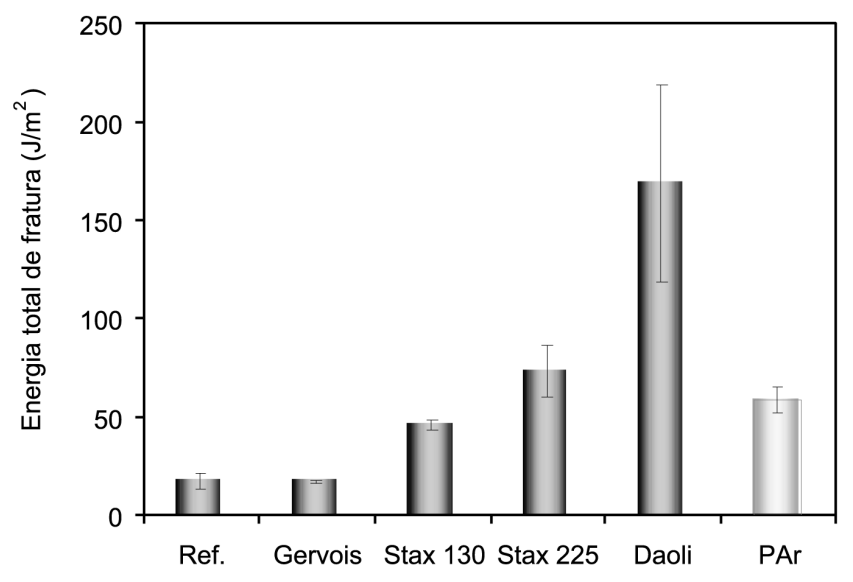

Figura 7: Energia total de fratura dos concretos com fibras metálicas ou poliaramida.

[Figure 7: Work-of-fracture of the refractory castables containing steel or polyaramid fibers.]

concreto não são suficientes para modificar sensivelmente sua resistência mecânica e módulo de elasticidade $[4,11,12]$.

Por outro lado, o comportamento mecânico do material após o início da propagação de trincas é bastante influenciado pela presença de fibras, como indica a energia total de fratura $\left(\gamma_{\text {wof }}\right)$ dos compósitos, mostrada na Fig. 7. A energia de fratura seguiu um comportamento crescente com relação ao comprimento médio das fibras metálicas adicionadas, embora o $\gamma_{\text {wof }}$ do concreto com as fibras Gervois tenha sido pouco alterado (Fig. 7). O $\gamma_{\text {wof }}$ do material contendo fibras metálicas Stax 130, Stax 225 e Daoli mostrou-se superior ao daquele contendo fibras de poliaramida, para o mesmo teor volumétrico [2]. O aumento relativo proporcionado pela fibra Daoli foi de cerca de $900 \%$, enquanto o obtido com a poliaramida, $250 \%$.

O aumento no consumo de energia após o início da propagação nos compósitos contendo fibras é ocasionado pelo fenômeno de ancoragem (bridging). Este efeito se deve principalmente à resistência à abertura da trinca e ao atrito 


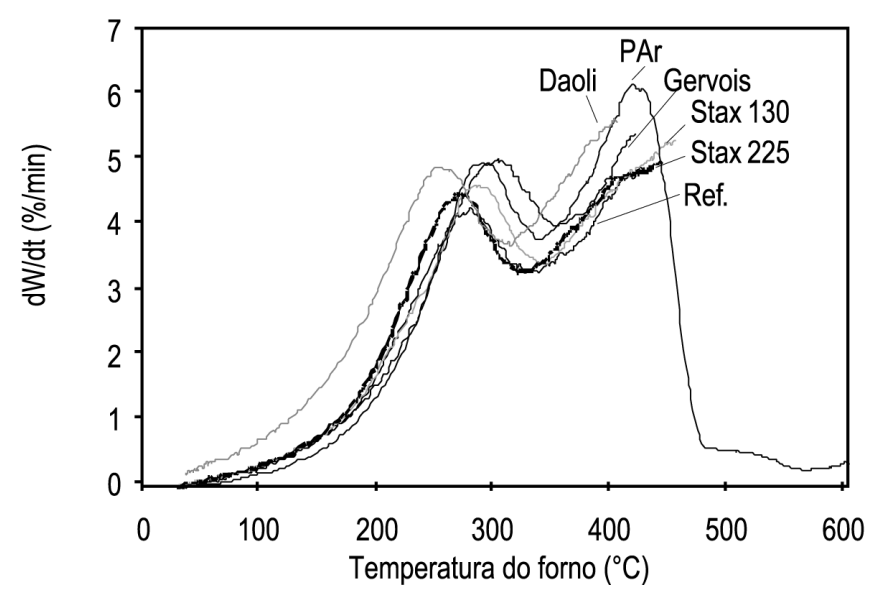

Figura 8: Taxa de secagem em função da temperatura do forno, para uma taxa de aquecimento de $20^{\circ} \mathrm{C} / \mathrm{min}$.

[Figure 8: Drying rate as a function of the furnace temperature, for a heating rate of $20^{\circ} \mathrm{C} / \mathrm{min}$.]

gerado para o arrancamento das fibras do concreto.

Devido aos baixos resultados de $\gamma_{\text {wof }}$ obtidos com a fibra Gervois, observa-se a existência de um comprimento mínimo a fim de que haja possibilidade de ancoragem da fibra na microestrutura do concreto, proporcionando, assim, a desejada dissipação de energia.

A análise termogravimétrica não revelou diferenças apreciáveis de taxa de perda de massa entre os materiais contendo fibras metálicas (Fig. 8), e em todos os casos, os corpos testados a $20^{\circ} \mathrm{C} / \mathrm{min}$ foram danificados.

Em contrapartida, o material contendo fibras de PAr sobreviveu ao aquecimento a $20^{\circ} \mathrm{C} / \mathrm{min}$, indicando a maior eficiência dessas fibras para o aumento da resistência à secagem e explosão.

A atuação mecânica das fibras no aumento da resistência ao dano durante a secagem (etapa de pressurização do vapor de água no material) está relacionada com o aumento da energia de fratura proporcionado ao concreto. Entretanto, para que a solicitação mecânica durante a secagem possa ativar o mecanismo de bridging é necessário que várias fibras estejam no caminho da trinca em formação. Desta forma, a distância entre as fibras deve ser suficientemente pequena e compatível com as dimensões das trincas na situação em questão.

A Fig. 9 mostra esquematicamente a microestrutura de um material com fibras de dois tamanhos distintos, mantendose constante seu teor volumétrico. As fibras de menores dimensões estarão presentes em maior número no material. Com isso, a distância média entre elas na microestrutura, $\mathrm{S}$, torna-se menor, e pode ser dada por

$$
S=2.4 \sqrt{\frac{\pi \cdot d^{2} \cdot I}{4 \cdot p}}
$$

em que d é o diâmetro da fibra, L é o seu comprimento,

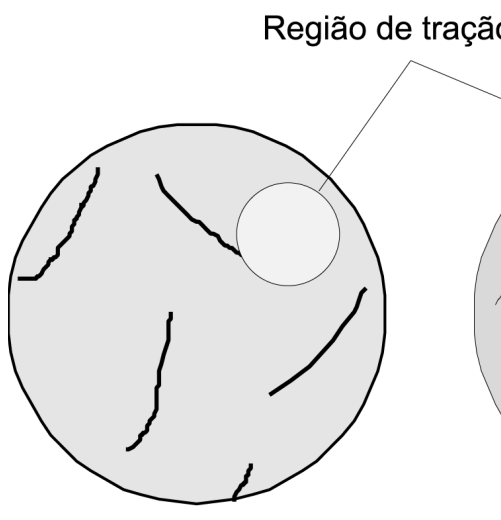

(a)

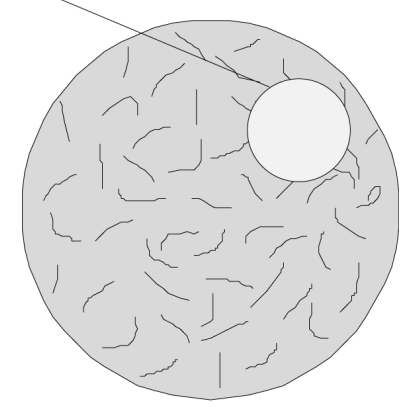

(b)
Figura 9: Representação esquemática de regiões pressurizadas em um concreto com fibras de (a) grandes e (b) pequenas dimensões, adicionadas no mesmo teor volumétrico.

[Figure 9: Representation of a pressurized region in the microstructure of a castable reinforced by (a) large and (b) small fibers, at the same volumetric content.]

Tabela IV - Distância entre as fibras nos concretos contendo $0,36 \%$-vol. de fibras metálicas ou de poliaramida.

[Table IV - Distance between fibers in the castables containing 0.36\%-vol. of steel or polyaramid fibers.]

\begin{tabular}{cc}
\hline & $\mathrm{S}(\mathrm{mm})$ \\
\hline Poliaramida & 0,17 \\
\hline Gervois & 0,35 \\
\hline Stax 130 & 0,92 \\
\hline Stax 225 & 1,56 \\
\hline Daoli & 7,98 \\
\hline
\end{tabular}

e p é o teor volumétrico percentual adicionado [13]. Vale lembrar que a Equação D provém de um modelo para fibras metálicas, desconsiderando o enovelamento típico de fibras com alta razão de aspecto e alta flexibilidade.

De acordo com a Equação D, a distância entre as fibras cresce na ordem PAr $<$ Gervois < Stax $130<$ Stax $225<$ Daoli, como mostra a Tabela IV.

No momento da secagem, o caminho médio percorrido por uma trinca em formação até ser interceptada por uma fibra corresponde à metade da distância entre as fibras.

De acordo com a teoria de Griffith, ao se propagar em condições de instabilidade, uma trinca adquire energia cinética crescente em função de seu comprimento. Para que a absorção dessa energia seja eficiente na interrupção da propagação é importante que as fibras interceptem a trinca quando a energia cinética ainda for baixa. Dessa forma, para a secagem, em que o comprimento máximo das trincas para que não haja explosão é muito pequeno, a presença de várias fibras distribuídas de forma mais homogênea gera um benefício maior ao material. 
Por esse motivo, as fibras de poliaramida foram as únicas a evitar a explosão no presente estudo. Os resultados evidenciam que as fibras metálicas de $25 \mathrm{~mm}$ de comprimento, eficientes para o aumento da resistência do material refratário ao choque térmico, ao impacto e à fadiga, não surtem efeito no aumento da resistência ao dano por secagem [14].

Se existirem no concreto regiões desprotegidas pela atuação mecânica das fibras, estas ficarão vulneráveis à ocorrência de dano localizado, podendo ser um ponto de início para a explosão.

\section{CONCLUSÕES}

Embora as fibras metálicas tenham provocado alterações bastante significativas principalmente na energia total de fratura do concreto, sobretudo quando comparada ao concreto com fibras de poliaramida, esse benefício não garantiu o aumento da resistência do material à explosão durante a secagem.

A pressurização ocorrida durante a secagem do concreto é localizada microestruturalmente na matriz do concreto. Tal característica gera a necessidade de fibras pouco distanciadas entre si, o que não é conseguido com fibras de volumes maiores, como as metálicas estudadas neste trabalho.

Os resultados indicam que os valores absolutos da resistência mecânica e da energia total de fratura não são os únicos parâmetros mecânicos que controlam a resistência do material ao dano durante a secagem. Três fatores principais devem ser levados em consideração durante a escolha de fibras para o reforço de concretos refratários, com vistas à secagem:

- A fibra deve possuir comprimento suficiente para garantir sua ancoragem na matriz, de forma que o consumo de energia para seu arrancamento seja considerável;

- O volume unitário da fibra deve fazer com que a distância entre elas seja compatível com a escala espacial das solicitações mecânicas;

- As características mecânicas do agente de reforço devem ser mantidas na temperatura em que as solicitações estarão atuando (no caso da secagem, entre 150 e $200{ }^{\circ} \mathrm{C}$ para a temperatura do corpo);

\section{AGRADECIMENTOS}

Os autores agradecem à FAPESP, Magnesita S.A. e ALCOA-Brasil pelo suporte; à Daoli Brasil-China, à Stax AG (Alemanha), à Gervois S.A. (França) e a DuPont AFS (Estados Unidos) pelo fornecimento das fibras utilizadas.

\section{REFERÊNCIAS}

[1] M. D. M. Innocentini, C. Ribeiro, R. Salomão, V. C. Pandolfelli, I. R. M. Bittencourt, Assessment of mass loss and permeability changes during the dewatering process of refractory castables containing polypropylene fibers, J. Am. Ceram. Soc. 85, 8 (2002) 2110-2112.

[2] C. M. Peret, R. Salomão, A. M. Zambon, V. C. Pandolfelli, Reforço mecânico por fibras poliméricas e seus efeitos na secagem de concretos refratários, Cerâmica 49, 312 (2003) 257-261.

[3] H. G. Jiang, J. A. Valdez, Y. T. Zhu, I. J. Beyerlein, T. C. lowe, The strength and toughness of cement reinforced with bone-shaped steel wires, Compos. Sci. Technol. 60, 9 (2000) 1753-1761.

[3] M. D. M. Innocentini, M. F. S. Miranda, F. A. Cardoso, V. C. Pandolfelli, Vaporization processes and pressure buildup during dewatering of dense refractory castables, J. Am. Ceram. Soc. 86, 9 (2003) 1500-1503.

[4] E. Cailleux, Microstructure et comportement thermomécanique d'un béton réfractaire renforcé par des fibres métalliques. Thèse (Doctorat, Sciences et Génie des Matériaux), École Nationale Supérieure des Mines de Paris, Paris (2001).

[5] R. G. Pileggi, V. C. Pandolfelli, A. E. M. Paiva, J. Gallo, Novel rheometer for refractory castables, Am. Ceram. Soc. Bull. 79, 1 (2000) 54-58.

[6] R. G. Pileggi, A. R. Studart, V. C. Pandolfelli, J. Gallo, How mixing affects the rheology of refractory castables, Part 1, Am. Ceram. Soc. Bull. 80, 6 (2001) 27-31.

[7] R. G. Pileggi, A. R. Studart, V. C. Pandolfelli, J. Gallo, How mixing affects the rheology of refractory castables, Part 2, Am. Ceram. Soc. Bull. 80, 7 (2001) 38-42.

[8] M. D. M. Innocentini, A. R. F. Pardo, V. C. Pandolfelli, Influence of air compressibility on the permeability evaluation of refractory castables, J. Am. Ceram. Soc. 83, 6 (2000)1536-1538.

[9] M. D. M. Innocentini, C. Ribeiro, J. Yamamoto, A. E. M. Paiva, V. C. Pandolfelli, L. R. M. Bittencourt, R. P. Rettore, Drying behavior of refractory castables, Am. Ceram. Soc. Bull. 80, 11 (2001) 47-56.

[10] R. Salomão, V. G. Domiciano, M. D. M. Innocentini, R. G. Pileggi, L. R. M. Bittencourt, V. C. Pandolfelli, Comportamento Reológico de Concretos Refratários Contendo Fibras Poliméricas. Anais do $46^{\circ}$ Congresso Brasileiro de Cerâmica, 2002, S. Paulo, Associação Brasileira de Cerâmica (2002) 1 CD-ROM.

[11] E. ABSI, (org.) Béton de fibres: synthèse dês études et recherches réalisées au CEBTP. Annalles de l'Institut Technique du Batîment et des Travaux Publics 250 (1994) 85-127.

[12] A. M. Pallière, (org.) Le béton de fibres métalliques: état actuel des connaissances. Annales de l'Institut Technique du Bâtiment et des Travaux Publics 515 (1993) 37-68.

[13] Pilbrico, Technology of Monolithic Refractories. Pilbrico Japan Company Ltd. (1984) 188-198.

[14] P. C. Tatnall, Shotcrete in Fires: Effects of Fibers on Explosive Spalling, Shotcrete, Fall (2002) 10-12.

(Rec. 07/07/2004, Ac. 18/02/2005) 